\title{
Friction Stir Welding of steels: AReview Paper
}

\author{
Kudzanayi Chiteka \\ (Production Engineering, Delhi Technological University, India)
}

\begin{abstract}
Friction Stir Welding (FSW) is a solid state joining process that can be applied to a number of materials including aluminium, magnesium, copper and steels. A number of researches have been conducted in Friction Stir Welding of steels and it is the focus of this paper to make a comprehensive review of the work that has been done. Ultra-low carbon steels, low carbon steels, medium carbon steels, high carbon steels and ultrahigh carbon steels have been considered and several aspects of FSW of steels have been outlined. These are tools, mechanical properties and microstructure. It was determined that carbon content, welding speed as well as rotational speed affect both mechanical properties and microstructure of the joint.
\end{abstract}

Key words: Friction Stir Welding, FSW parameters, FSW tools, Mechanical properties, Microstructure.

\section{Introduction}

The technique of Friction Stir Welding (FSW) was developed at The Welding Institute (TWI) in 1991 as a method to join non-ferrous materials like aluminium and magnesium. Due to difficulties of high temperature involved in joining of steel which has an effect of rapid softening and wearing of the tool, this process was not so popular in joining of steel and other hard materials like titanium. Of late, it has been discovered that if FSW is applied to steels, the mechanical properties were close to the base materials (Choi et al.2011) [1].There have been a discovery of suitable tool material such as Tungsten Carbide (WC) and Polycrystalline cubic boron nitride (PCBN) for FSW tools which can join hard alloys such as steels and Ti alloys. Because of such discoveries, the joining of steels became increasingly popular (Rai et al. 2011)[2].Fujii et al. (2006)[3], noted that the use of FSW process had the advantage that material is not melted and hence grain growth cannot occur when this method is applied thus it has been shown that the process of FSW is a solid state process which does not involve the melting of the materials being joined. The residual stresses and distortion in FSW welds are generally lower than those found in fusion welds. The associated lower energy input in FSW tends to minimise grain growth in the Heat Affected Zone (HAZ) thus limiting distortion and residual stresses. FSW being a solid state process eliminates the problems associated with hydrogen cracking in steels (Lienert et al. 2003) [4].

In FSW process, a shouldered tool with a probe is rotated and traversed along the joint line of the two materials being joined. It is that rotation which cause frictional heating that softens and plasticise the work material resulting in joining. Yousif et al. (2008) [4] made a remarkable statement when he said that FSW is rather a combined effect of forging and extrusion and not a true welding process in actual fact.

\section{Successfully welded steels}

Lienert et al. (2003) [5] undertook a feasibility study on the FSW of mild steel (hot-rolled AISI 1018 steel plates) $6.35 \mathrm{~mm}$ thick with welding done parallel to the rolling direction. The research yielded defect free welds with traverse speeds ranging from 0.43 to $1.68 \mathrm{~mm} / \mathrm{s}$ at speeds varying from 450 to $650 \mathrm{rpm}$. This process was done in the presents of a shielding gas. Molybdenum-based and tungsten-based alloy tools were used. In this undertaking a peak surface temperature of $1000{ }^{\circ} \mathrm{C}$ was attained and also failure was not in the joint but rather in the base metal implying a stronger joint. A tensile strength of $476 \mathrm{MPa}$ was attained which surpassed that of the base metal of $463 \mathrm{MPa}$.

Cho et al. (2012) [6] studied the microstructural evolution in friction stir welding of high-strength API X100 grade linepipe steel $10 \mathrm{~mm}$ on thickness using a PCBN tool with $16 \mathrm{~mm}$ shoulder diameter and $4 \mathrm{~mm}$ pin diameter in an inert gas environment. A rotation speed of $450 \mathrm{rpm}$ and a traverse speed of $127 \mathrm{~mm} / \mathrm{min}$ were used. Acicular-shaped bainitic ferrites were formed by the phase transformation in most parts of the stir zone (SZ). In the thermo-mechanically affected zone (TMAZ), a fine-grained microstructure was observed and this was attributed to continuous dynamic recrystallization. There was higher hardness in the SZ compared to other regions.

Sato et al. (2007) [7] analysed the FSW of ultrahigh carbon steel $2.3 \mathrm{~mm}$ thickness consisting of a (ferrite + cementite) duplex structure. They used a polycrystalline cubic boron nitride (PCBN) tool and defectfree welds were produced successfully at rotational speeds between 400 and $800 \mathrm{rpm}$. When all welds were examined, there was no large difference in microstructure and hardness. It was found that the hardness profile and microstructural distribution in ultra-high carbon steels are not dependent on the rotational speed. 
Jafarzadegan et al. (2013) [8] studied the FSW of 304 stainless steel and st37 steel at $600 \mathrm{rpm}$ and $50 \mathrm{~mm} / \mathrm{min}$ traverse speed. A refined grain structure was identified in the SZ and this increased the hardness and tensile strength in the SZ.

Reynolds et al. (2003)[9] in the FSW study of the SZ of 304L $3.2 \mathrm{~mm}$ thick recorded grain refinement in stainless steel and also there was high tensile properties and hardness. The yield strength obtained was higher than that of the base metal (BM) attaining a value of $430 \mathrm{MPa}$ at $300 \mathrm{rpm}$ compared to $295 \mathrm{MPa}$ of the base metal. A tensile strength of $735 \mathrm{MPa}$ was attained at that same speed compared to $667 \mathrm{MPa}$ for the base metal. In another study using same material Park et al. (2003) [10] produced comparable results.

Fujii et al. (2006)[3] examined the effect of carbon content in different carbon steels (IF steel, S12C, S35C) in FSW, and analysed the effect of peak temperature on the mechanical and microstructural properties. There was a uniform grain structure in the SZ composed of equiaxed grains with sub-structures. The grain sizes at the SZ centre welded under lowest and highest heat input conditions were 5 and $6 \mu \mathrm{m}$ respectively which are far smaller compared to $24 \mu \mathrm{m}$ for the base metal.

Lakshminarayanan and Balasubramanian (2010)[11] studied the Microstructure and mechanical characterization of friction stir welded 409M ferritic stainless steel joint produced by FSW. Defect-free joints were produced at a welding speed of $50 \mathrm{~mm} / \mathrm{min}$ and rotation speed of $1000 \mathrm{rpm}$. Overmatching of the joint relative to the $\mathrm{BM}$ was observed in tensile tests. Acceptable ductility and impact toughness were also witnessed.

FSW of SK5 (0.84 wt\% C) steel by Choi et al. (2011) [1] resulted in less martensite formation when a gas torch was used in one set of experiments and not used in another. However, the tensile strength results were similar to those of the BM.Cui et al. (2007) [12] recorded successful joining of high carbon steel S70C. The hardness of the joint was higher than the BM. Chung et al. (2010) [13] also successfully joined high carbon steel ( 0.85 mass $\%$ C, AISI-1080) at a temperature below $\mathrm{A}_{1}$ to exclude martensite formation in the joint. Defect-free joints have been produced in this process as well.

\section{Joining of steels to other metals}

Steels have also been joined to other types of metals which include aluminium as studied by Wanatabe et al.(2006) [14].A butt weld was produced between aluminium A5083 ( $\mathrm{Al}-4.5$ mass $\% \mathrm{Mg}-0.5$ mass\% MN) and mild steel plates (SS400) $2 \mathrm{~mm}$ in thickness. The joining was successful and easily attained. A tensile strength $86 \%$ of that of the base metal was achieved. The upper part of steel/aluminum interface had a small amount of intermetallic compounds formed but this was not the case in the middle and bottom parts of the interface. These regions with intermetallic compounds were the fracture paths in the joint. The aluminium alloy matrix had scattered fragments of steel.Dehghani et al. (2012) [15], conducted FSW on 3mm mild steel (St52) sheet and a cold rolled 3003-H18 aluminum alloy sheet. Defect-free joints were produced in this study. Maximum UTS of $96 \mathrm{MPa}$ was attained on the joint and this was higher than the aluminium BM.In another research, Coelho et al (2012) [16] successfully joined DP600 and HC260LA HSS plates to AA6181-T4 Al alloy by FSW. Defect-free joints were produced in this research and UTS tests gave $80 \%$ joint strength compared to the aluminium BM.Joining of steel to aluminium has proved to be feasible with joint strength attaining about $80 \%$ of the aluminium base metals.

\section{Process parameters}

Like in any other FSW process, the most significant process parameters in FSW of steels include welding speed, spindle speed, tool geometry and the axial force applied.Microstructural evolution is controlled by total strain, strain rate and the temperatures attained in the welding process (Lienert et al, 2003) [5].

Mishra and Ma (2005) [17] stated that tool geometry is the most influential parameter which plays an important part in material flow in FSW process. Tool geometry determines the welding speed at which FSW can be performed.Threaded cylindrical pins have been found to be very popular with FSW. It has also been noted by Mishra and Ma (2005) [17] that higher tool rotation results in higher temperatures due to higher friction heating. This will result in intense stirring and mixing of material.

Fujii et al. (2006) [3], studied the effect of carbon content in the FSW of IF, S12C and S35C. It was proven that the welding conditions had a notable effect to microstructures and mechanical properties of the carbon steel joints. As the carbon content increases, the higher are the chances of phase transformations, as such; the microstructure becomes highly dependent on the parameters used. These parameters are in particular, welding speed and rotational speed which determine the temperature output which in turn determine phase transformation and thus martensite formation in carbon steels.In certain instances, a shielding gas can be used to prevent oxidation at high temperatures involved Chung et al. (2010) [13].

\section{Microstructural Characterisation}

Fujii et al (2006) [5],noted that FSW in steels generally has a grain refinement in the stir zone of the carbon steel just similar to Al alloys. On top of that, complex phase transformations also occur in the FSW 
process.Four regions have been identified in FSW and these correspond to Nugget Zone (stir zone), Thermomechanically Affected Zone, Heat Affected Zone (HAZ) and Base Metal. According to the studies done on high-strength API X100 grade linepipe steel by Cho et al (2012) [6],there is grain growth in HAZ due to high heat levels attained in this zone. The microstructure of this zone only differs with the BM in grain size. Like the SZ, the TMAZ has a fine grained microstructure due to continuous dynamic recrystallization.In SZ there is a significant difference in the structure morphology, texture and misorientation-angle distribution. Unlike the other three zones, the SZ is difficult to analyse as stated by Lienert et al. (2003) [5]in FSW of mild steel.The effects of thermo-mechanical cycle are both considered when giving an analysis of this zone in relation to microstructural development. Lienert et al. (2003) [5] associated this zone as undergoing a hot working process. Temperatures in this zone were said to exceed $1100^{\circ} \mathrm{C}$ and strain rates also exceeding 1 . Restorative processes which include dynamic recovery, dynamic recrystallization, and metadynamic recrystallization tend to operate individually or as a combination during the hot working process.

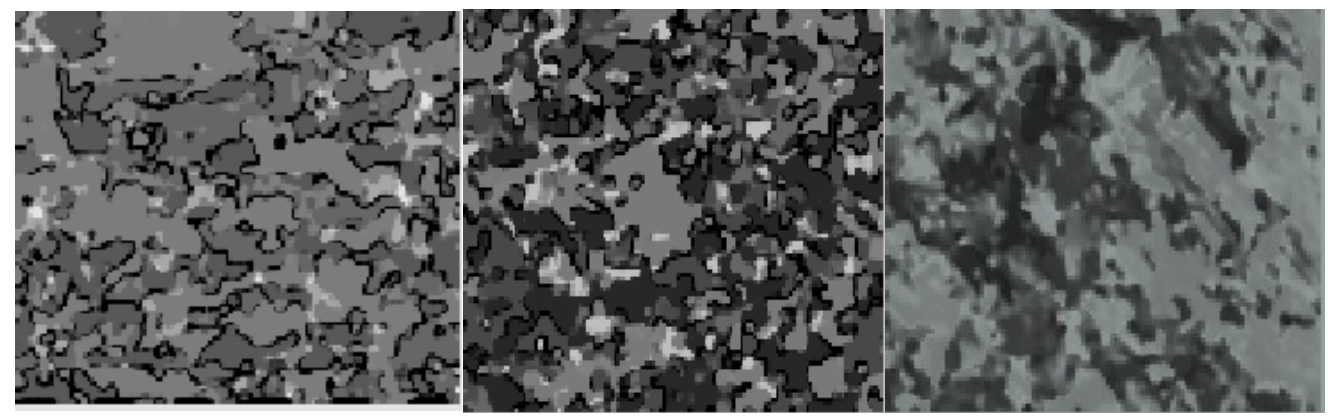

Figure 1. HAZ, TMAZ and SZ of high strength linepipe steel (Cho et al. 2012) [6].

Fujii et al (2006)[3], when they analysed interstitial free (IF), S12C (low carbon Steel) and S35C (medium carbon steel) observed that there was a uniform grain structure in the SZ composed of equiaxed grains with substructures. The grain sizes at the SZ centre welded under lowest and highest heat input conditions were 5 and $6 \mu \mathrm{m}$ respectively which are far smaller compared to $24 \mu \mathrm{m}$ for the BM.The entire IF steel FSW welds were done in the ferrite single-phase region and there was no phase transformation but heavy deformation and recrystallization was observed. Theydiscovered that ferrite-pearlite structures with a limited amount of pearlite were observed under all the welding conditions in the FSW of S12C and they noted that the total amount of carbides was very small. The medium carbon steel, S35C in almost all welding conditions, the SZ contained the ferrite-pearlite structures.
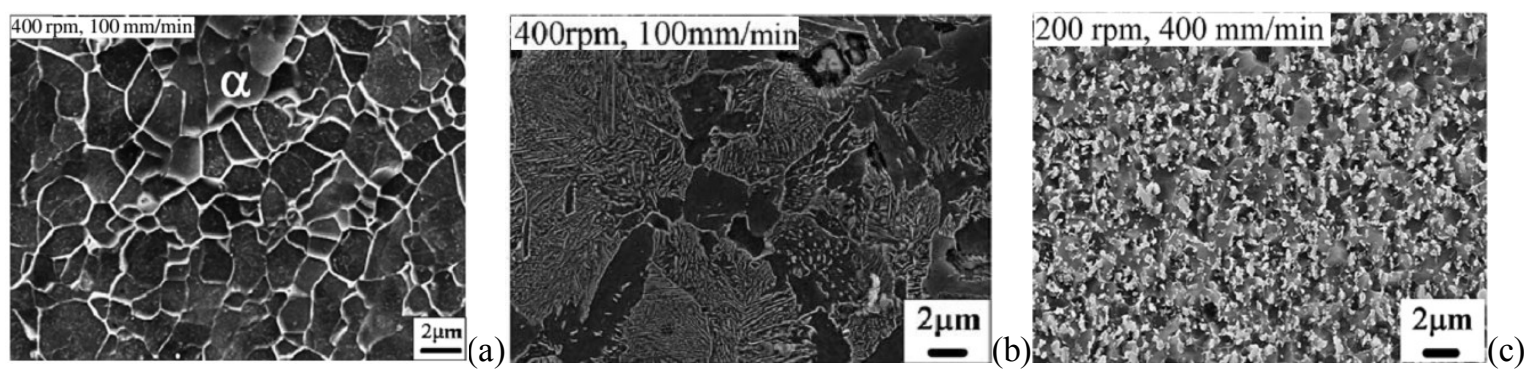

Figure 2. Different microstructures of: (a) S12C (b) S35C, Fujii et al. (2006) [3], (c) S70C, Cui et al. (2007)

[12].

\section{Mechanical properties}

Experiments which have been done on FSW of mild steel by Lienert et al (2003) [5] have revealed tensile properties very close to those of the BM. They attained yield strength $331 \mathrm{MPa}$ against $310 \mathrm{MPa}$ for the $\mathrm{BM}$ and a tensile strength of $476 \mathrm{MPa}$ against $463 \mathrm{Mpa}$ for the BM. The hardness values ranged from 155 to $175 \mathrm{VHN}$ compared to $135 \mathrm{VHN}$ for the BM. Bending tests at $180^{\circ}$ revealed tensile strain of $15 \%$ at the outer fibre.Fujii et al (2006)[3], in the study of the effect of carbon content in the FSW of IF, S12C and S35C noted that, welding speed increases the strength of the $\mathrm{S} 12 \mathrm{C}$ joints.Mechanical properties of steel joints produced by FSW have improved mechanical properties compared to the BM.The weld regions generally possess higher hardness although the hardness profile depends on the steel type and the welding conditions.

Fujii et al. [18] in the FSW of interstitial free steel (IF steel: $20 \mathrm{ppmC}$ ), and ultrafine grained steel discovered that there was no significant change in microstructure and mechanical properties due to the ultra-low carbon content. However this is not the situation with $\mathrm{S} 35 \mathrm{C}$ which is a medium carbon steel. 

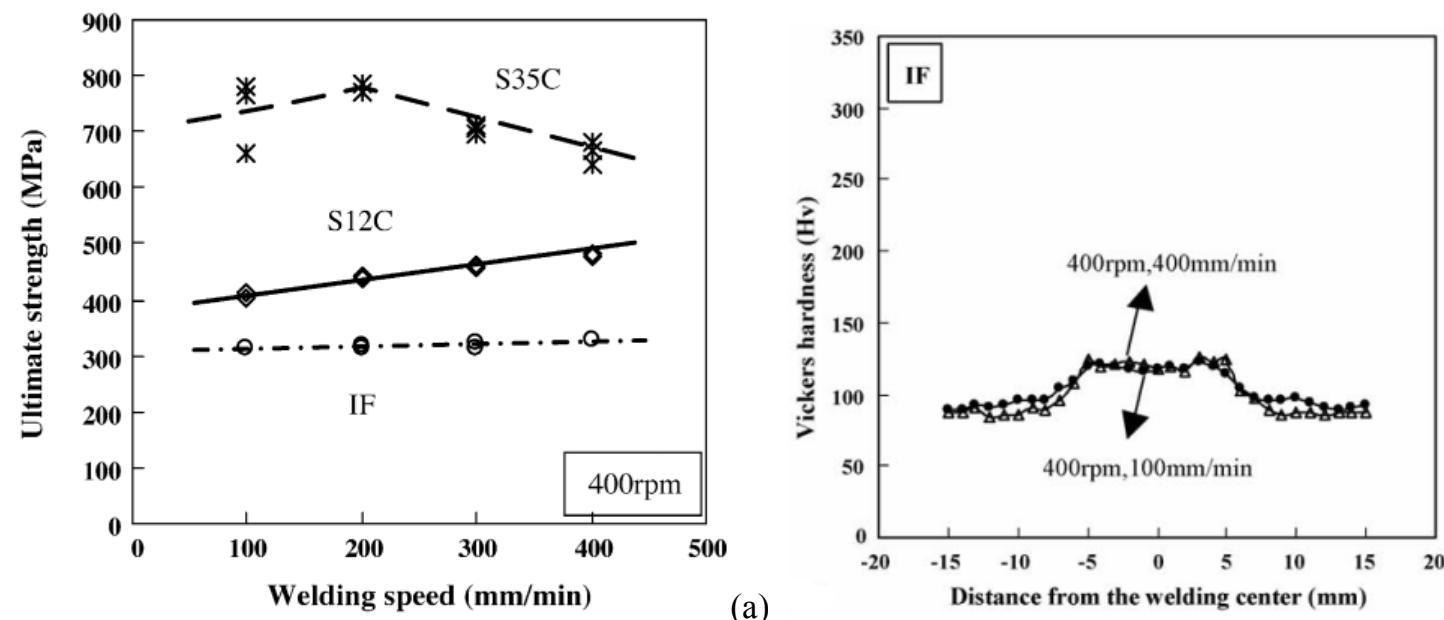

(a)

Figure 3. The relationship between welding speed and ultimate strength in IF, S12C and S35C and hardness of IF steel, Fujii et al. (2006) [3]
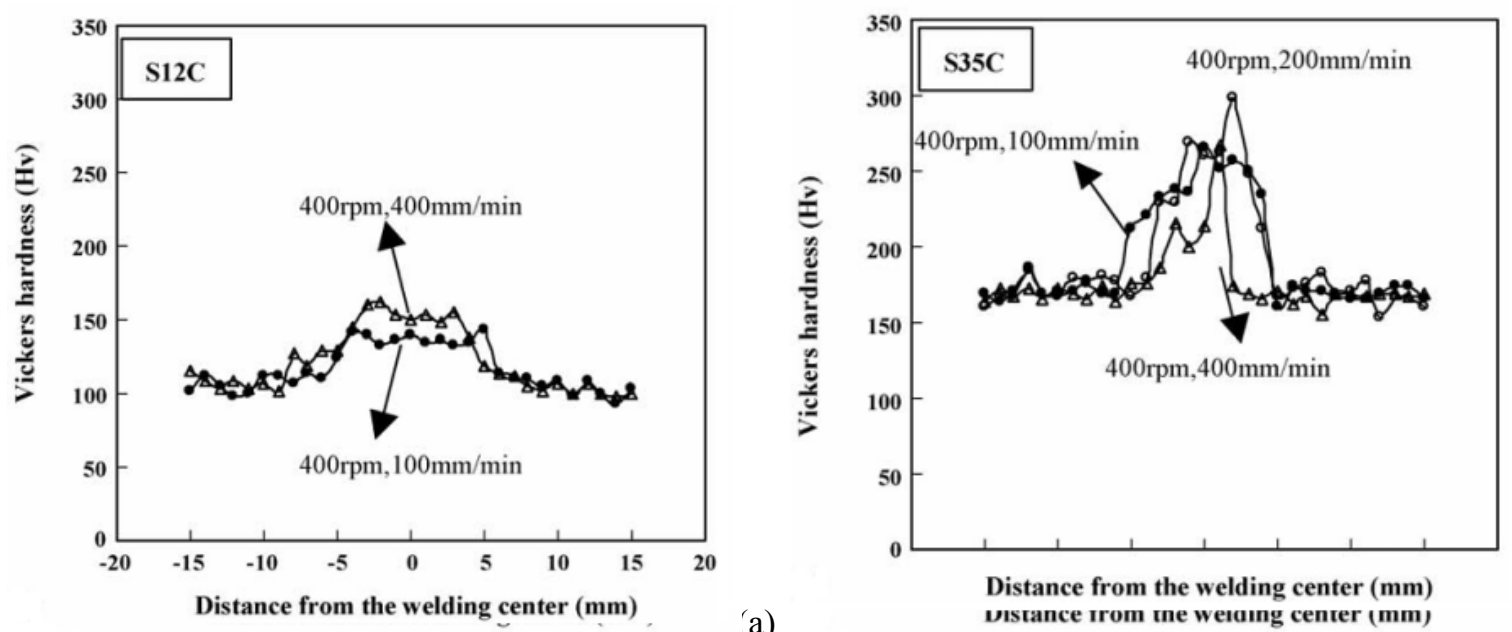

Figure 4. Hardness of IF steel, S12C and S35C, Fujii et al. (2006) [3]

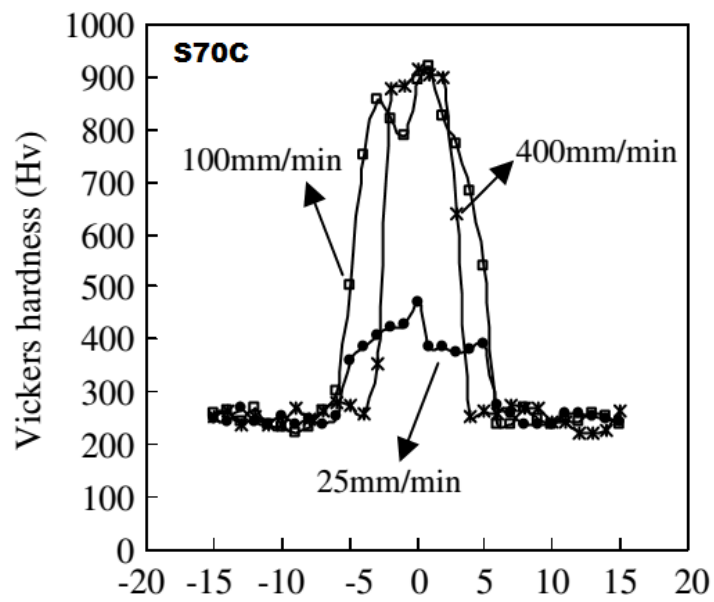

Distance from the welding center $(\mathrm{mm})$

Figure 5. Hardness of S70C, Cui et al (2007) [12]

\section{Tools used in FSW of steels}

Although there is rapid wear of the tool, this does not affect the tensile strength of the weld joint. However, tool life has remained a major concern.It has been noted that the plunging stage has the greatest amount of tool wear. This has been attributed to high loads and the cold state of the work during plunging. Some 
methods have been devised so that tool wear during plunging is minimised. It has been found that a partial solution can be found by preheating the plunge area, slow plunge rate, partial penetration and partial diameter hole (Lienert et al, 2003) [5].WC-based materials have been used in the joining of high carbon steels, Cui et al. (2007) [12].WC-based tools are strong at elevated temperatures. This tool material has excellent toughness and good thermal conductivity at temperatures below $\mathrm{A}_{1}$, Chung et al. (2010) [13].The joining of steels requires tools which can operate at elevated temperatures but still maintains high strength and hardness. Such materials include PCBN, Rai et al. (2011) [2]. This material can weld very hard materials including titanium. PCBN has a low coefficient of friction.

\section{Conclusion}

Very low carbon steels undergo fewer changes in comparison to the microstructures and mechanical properties of the carbon steels which are greatly affected by the welding parameters. Temperatures as well as carbon content have a huge bearing on the microstructure. High welding speed yields a higher joint hardness. The highest hardness that can be attained is three times as much as the BM, Cui et al. (2007) [12].Higher rotation speed in carbon steels also causes phase transformation which will have a strong effect to the mechanical properties of the joint.

FSW allows adjustments to be made so as to obtain a sound joint in steels. This can be attained by decreasing the peak temperature to below $\mathrm{A}_{1}$ and also decrease the cooling rate to less than the lower critical cooling rate. By so doing, FSW allows control of the cooling rate and peak temperature unlike other welding methods.

Friction Stir Welding has a grain refining effect in the SZ of carbon steels. Mechanical properties are also improved compared to the BM.

When FSW has been performed under appropriate conditions, joining of high carbon steels without transformation can be attained and due to the homogeneous microstructure in the joints, they have improved ductility.

In ultra-high carbon steel, the effect of the rotational speed on the hardness profile is negligible, and the microstructural distribution is also hardly dependent on the rotational speed,Sato et al. (2012) [7].

\section{References}

[1] Don-Hyun Choi, Chang-Yong Lee, Byung-Wook Ahn, Jung-Hyun Choi, Yun-Mo Yeon, Keun Song, Seung-Gab Hong, Won-Bae Lee, Ki-Bong Kang and Seung-Boo Jung, Hybrid Friction Stir Welding of High-carbon Steel, J. Mater. Sci. Technol., 2011,27(2), $127-130$.

[2] R. Rai, A. De, H. K. D. H. Bhadeshia and T. DebRoy, Review: friction stir welding tools, Science and Technology of Welding and Joining, 2011 VOL16 NO4 325.

[3] Hidetoshi Fujii, Ling Cui, Nobuhiro Tsuji, Masakatsu Maeda, Kazuhiro Nakata and Kiyoshi Nogi, Friction stir welding of carbon steels, Materials Science and Engineering, A 429 (2006) 50-57.

[4] Y. K. Yousif, K. M. Daws and B. I. Kazem, Prediction of Friction Stir Welding Characteristic Using Neural Network, Jordan Journal of Mechanical and Industrial Engineering, Vol. 2, No. 3 (2008) $151-155$.

[5] T. J. Lienert, W. L. Stellwag, Jr., B. B. Grimmett and R. W. Warke, Friction Stir Welding Studies on Mild Steel, Supplement To The Welding Journal, 2003.

[6] Hoon-Hwe Cho, Suk Hoon Kang, Sung-Hwan Kim, Kyu Hwan Oh, Heung Ju Kim, Woong-Seong Chang and Heung Nam Han, Microstructural evolution in friction stir welding of high-strength linepipe steel, Materials and Design 34 (2012) 258-267.

[7] Y.S. Sato, H. Yamanoi, H. Kokawa and T. Furuhara, Microstructural evolution of ultrahigh carbon steel during friction stir welding, Scripta Materialia 57 (2007) 557-560.

[8] M. Jafarzadegan, A. Abdollah-zadeh, A.H. Feng, T. Saeid, J. Shen and H. Assadi, Microstructure and Mechanical Properties of a Dissimilar Friction Stir Weld between Austenitic Stainless Steel and Low Carbon Steel, J. Mater. Sci. Technol., 2013, 29(4), 367372 .

[9] A.P. Reynolds, Wei Tang, T. Gnaupel Herold and H. Prask, Structure, properties, and residual stress of 304L stainless steel friction stir welds, Scripta Materialia 48 (2003) 1289-1294.

[10] Seung Hwan C. Park, Yutaka S. Sato, Hiroyuki Kokawa, Kazutaka Okamoto, Satoshi Hirano and Masahisa Inagaki, Rapid formation of the sigma phase in 304 stainless steel during friction stir welding, Scripta Materialia 49 (2003) 1175-1180.

[11] A.K. Lakshminarayanan and V. Balasubramanian, An assessment of microstructure, hardness, tensile and impact strength of friction stir welded ferritic stainless steel joints, Materials and Design 31 (2010) 4592-4600

[12] Ling Cui, Hidetoshi Fujii, Nobuhiro Tsuji and Kiyoshi Nogi, Friction stir welding of a high carbon steel, Scripta Materialia 56 (2007) 637-640.

[13] Y.D. Chung, H. Fujii, R. Ueji and N. Tsuji, Friction stir welding of high carbon steel with excellent toughness and ductility, Scripta Materialia 63 (2010) 223-226.

[14] Takehiko Watanabe, Hirofumi Takayama and Atsushi Yanagisawa, Joining of aluminum alloy to steel by friction stir welding, Journal of Materials Processing Technology 178 (2006) 342-349.

[15] M. Dehghani, S. A. A. Akbari Mousavi and A. Amadeh, Effects of welding parameters and tool geometry on properties of 3003H18 aluminum alloy to mild steel friction stir weld, Trans. Nonferrous Met. Soc. China 23(2013) 1957-1965.

[16] R.S. Coelho, A. Kostka, J.F. dos Santos and A. Kaysser Pyzalla, Friction-stir dissimilar welding of aluminium alloy to high strength steels: Mechanical properties and their relation to microstructure, Materials Science \& Engineering A 556 (2012) $175-183$.

[17] R.S. Mishra and Z.Y. Ma, Friction stir welding and processing, Materials Science and Engineering R 50 (2005) 1-78.

[18] Hidetoshi Fujii, Ueji Rintaro, Cui Ring, Nakata Kazuhiro and Kiyoshi Nogi, Friction stir welding of ultrafine grained IF and carbon steels, Transactions of JWRI, Vol. 35 (2006), No. 1. 